\title{
BG03
}

\section{Possible Pitfalls of LWD Images}

\section{A.K. Thorsen* (Baker Hughes Norge AS)}

\section{SUMMARY}

During the last decade there has been a development in the usage of images obtained during drilling. In general there are three different measurements utilized to obtain the measurements, Gamma, density and resistivity, all with different qualities and usages.

Going back to the initial phase of logging while drilling (LWD) technologies, the initial view was to ensure usage of data in real-time and take action based upon the real-time data obtained. 


\section{EAGE}

\section{Introduction}

During the last decade there has been a development in the usage of images obtained during drilling. In general there are three different measurements utilized to obtain the measurements, Gamma, density and resistivity, all with different qualities and usages.

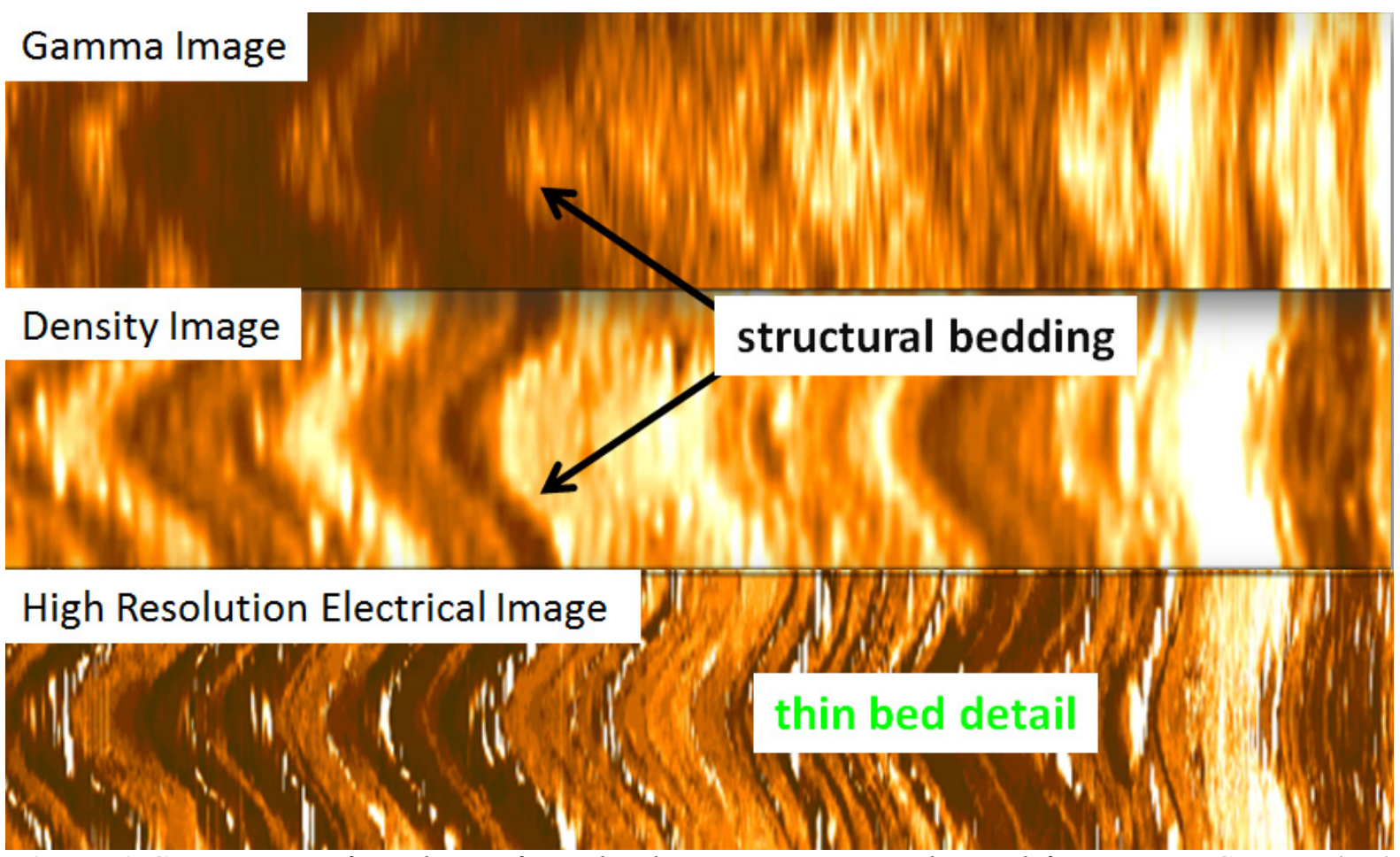

Figure 1 Comparison of resolution from the three main images obtained from LWD; Gamma (top) Density (middle) High resolution electrical (bottom). The increase in resolution from the gamma image through the density and electrical images are well defined, and hence the usage from pure structural bedding interpretation to a full sedimentological analysis.

\section{Overview}

Going back to the initial phase of logging while drilling (LWD) technologies, the initial view was to ensure usage of data in real-time and take action based upon the real-time data obtained.

In the case of using LWD images, a thorough understanding of the environment of where the data are obtained and how the impact of the physical properties of the drilling process is influencing the result and accuracy of the result and the interpretation. Initial gamma images where based upon 4 cures, up/down/left/right, and made for the sole purpose of determining the direction of an approaching bed with different shale content. The gamma reading was then followed by a more precise and improved image based on density with the same target, to define direction of an approaching layer, this time based on a difference the bulk density. Following these "low" resolution images, resistivity images were developed for conductive drilling fluids with an extremely high resolution for a full blown sedimentological evaluation.

This presentation will go through the effects from the drilling process affecting the different LWD images generated and to which degree. The main aspects are related to depth control of the down hole measurement, actual physical aspects of the drill string and how the vibration pattern will influence on the end result. 


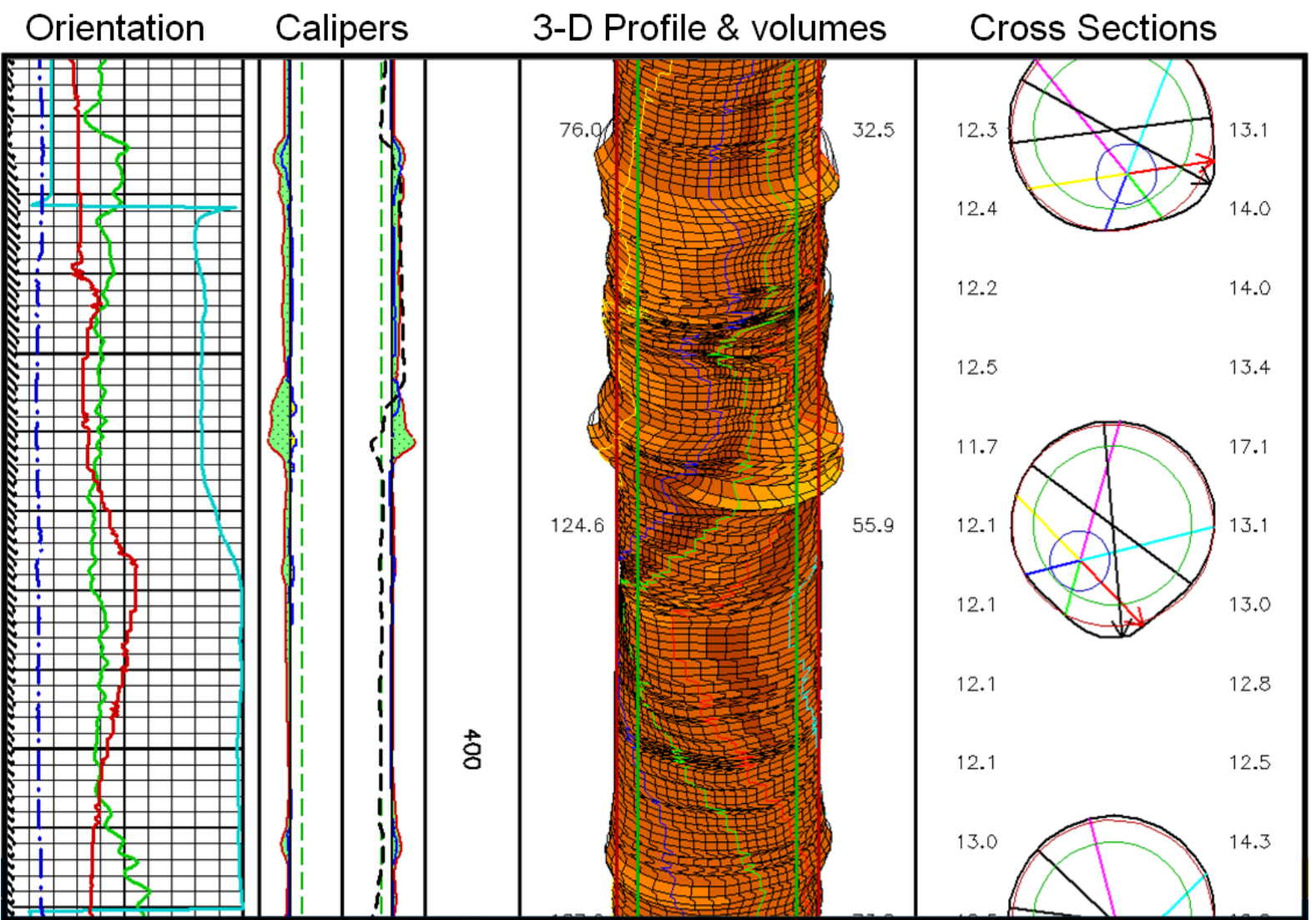

Figure 2 To fully understand the image interpretation a caliper log obtained at the same time as the image log is vital in the interpretation phase. Time is a vital factor in wellbore stability understanding. Combining image logs from LWD with caliper logs obtained at a later time might lead to a biased interpretation.

Analyzing data from the three different image types allow a comparison on data acquired at more or less the same time, and an evaluation of the similarities and difference, including interpretation of why these effects are occurring.

\section{Conclusions}

To fully understand the difference between the two, other parameters obtained during the drilling process will be included in the overall interpretation and used to fully understand the overall process. Additional data are typically obtained during the drilling process, although not commonly incorporated in the interpretation, this is related to differences in drilling parameters at surface and downhole and the part the well trajectory and mud properties are playing in the overall process.

\section{References}

Hartmann, A., Akimov, O., Morris, S. and Fulda, C. [2012] Improving Real-Time Image-Data Quality with a Telemetry Model. SPE Drilling \& Completion, September, 382-391.

Martin, S.J., Thomas, M.G., Hammons, S.D. and Wilke, N. [2013] Real-Time Azimuthal Gamma and Resistivity LWD Data Used to Navigate Complex Unconventional Reservoir - Western US Land. SPE Asia Pacific Oil \& Gas Conference and Exhibition. Jakarta, Indonesia, 22-24 October 2013. SPE paper number 165923. 
Dashevskiy, D., Dahl, T., Brooks, A.G., Zurcher, D., Lofts, J.C. and Dankers, S. [2008] Dynamic Depth Correction to Reduce Depth Uncertainty and Improve MWD/LWD Log Quality. SPE Drilling \& Completion, March, 13-22. 